\title{
Amplitude Control in a van der Pol-Type Self-Excited AFM Microcantilever*
}

\author{
Masaharu KURODA**, Hiroshi YABUNO ${ }^{* * *}$, Keiichi HAYASHI ${ }^{\dagger}$ and \\ Kiwamu ASHIDA ${ }^{\dagger \dagger}$ \\ ** National Institute of Advanced Industrial Science and Technology (AIST) \\ 1-2-1 Namiki, Tsukuba, Ibaraki, 305-8564, Japan \\ E-mail: m-kuroda@aist.go.jp \\ *** Graduate School of Systems Information Engineering, University of Tsukuba \\ 1-1-1 Tennoudai, Tsukuba, Ibaraki, 305-8573, Japan \\ E-mail: yabuno@esys.tsukuba.ac.jp \\ Graduate School of Systems Information Engineering, University of Tsukuba \\ 1-1-1 Tennoudai, Tsukuba, Ibaraki, 305-8573, Japan \\ E-mail: someya@aosuna.esys.tsukuba.ac.jp \\ ${ }^{\dagger}$ National Institute of Advanced Industrial Science and Technology (AIST) \\ 1-2-1 Namiki, Tsukuba, Ibaraki, 305-8564, Japan \\ E-mail: takeshi-kobayashi@aist.go.jp
}

\begin{abstract}
Usage of self-excitation as an excitation method for a cantilever probe in atomic force microscopy (AFM) has been proposed to improve the low quality factor Q in liquid environments. To realize non-contact mode AFM, it is necessary to reduce the amplitude of the self-excited cantilever probe. For this study, the self-excited oscillation of the cantilever probe is generated by the angular velocity feedback. In addition, the small steady state amplitude is achieved using nonlinear feedback proportional to the squared deflection angle and the angular velocity. Regarding the microcantilever probe as a microcantilever beam, we present the equation of motion, which incorporates the geometrical nonlinear effect. The averaged equation is derived by applying the method of multiple scales and the bifurcation diagram is described theoretically. Results clarify that increasing the nonlinear feedback gain can reduce the cantilever-probe amplitude. Using an AFM that we produced, we demonstrate the nonlinear dynamics of a "van der Pol" type of self-excited cantilever. The steady state amplitude of the self-excited oscillation was $8 \mathrm{~nm}$.
\end{abstract}

Key words : Nonlinear Vibration, Nonlinear Control, Self-Excited Vibration, Atomic Force Microscopy, Cantilever, van der Pol Oscillator

\section{Introduction}

Recently, atomic force microscopy (AFM) has become increasingly useful for observing biological samples in liquid environments ${ }^{(1)}$. Proper equipment for AFM enables observation of the surface morphology of small samples using a microcantilever with a probe on its free end. Changes in the distance between the sample and the probe are detectable based on the fact that atomic force acting between the surface and the probe alters the equivalent eigenfrequency of the cantilever ${ }^{(2)}$. We face difficulty in measuring the eigenfrequency using the conventional external excitation method because of the drastic decrease in the Q-factor of the cantilever that occurs because of viscous damping when we use AFM in liquid.

On the other hand, an application of self-excited oscillation is proposed as a method to measure the cantilever eigenfrequency in a high-viscosity liquid environment ${ }^{(3)(4)(5)}$. Especially as noted in ref. (5), although a DNA molecule was observed successfully, no countermeasures had yet been developed for vibration-amplitude control of the probe-cantilever.

*Received 15 Dec., 2007 (No.T2-07-0312) Japanese Original: Trans. Jpn. Soc. Mech. Eng., Vol.73, No.732, C (2007), pp.2225-2231 (Received 4 Apr., 2007) [DOI: 10.1299/jsdd.2.886] 


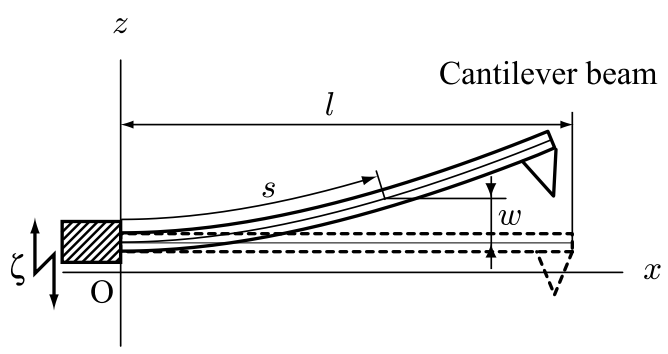

Fig. 1 Analytical model

Therefore, it is probable that the probe-cantilever stops when it is oscillating with small amplitude. A certain level of large-oscillation amplitude is necessary to keep the oscillation from stopping. However, such an oscillating probe-cantilever would then tend to suffer strong collisions with the specimen surface. Consequently, their method presents difficulties when stably observing objects such as biological samples, which are easily deformed or broken. The frequency of self-excited oscillation always coincides with the eigenfrequency. For that reason, it can detect the eigenfrequency, even in liquid and other environments. However, it is generally unavoidable that the amplitude of the cantilever in the self-excited oscillation condition increases over time. Therefore, to use self-excited oscillation to observe some materials, such as biological specimens that have soft and irregular surfaces which might be easily deformed by the slightest impact, a special technique is necessary to suppress, maintain, and limit the vibration amplitude of the cantilever and thereby prevent it from damaging the sample surface.

To achieve such a sufficiently small and steady vibration amplitude of the cantilever used in this study, we produced a microcantilever with vibration characteristics that are equivalent to van der Pol-type self-excited oscillation using linear feedback and nonlinear feedback ${ }^{(6)(7)}$. Van der Pol-type self-excited oscillation is well known to have steady state vibration with finite amplitude because of its nonlinearity ${ }^{(8)}$.

In other words, this amplitude characteristic ${ }^{(9)(10)}$ enables us to control the probe cantilever amplitude in a resonance condition; thereby the problem arising from the contact between the cantilever and the measurement sample can be resolved. For this study, we produced a prototype AFM to demonstrate the capability to generate steady self-excited oscillation with sufficiently small amplitude that it can realize AFM function during experiments.

\section{Dynamics of the probe cantilever in van der Pol type self-excited oscillation}

\subsection{Analytical model and equation of motion}

Assume an AFM probe cantilever as an Euler-Bernoulli beam to derive its equation of motion (EOM). An analytical model of the cantilever is shown in Fig. 1. Set the $x$-axis in the longitudinal direction of the cantilever and the $z$-axis in the deflection direction of the cantilever. The cantilever is expected to move in the $z-x$ plane. The cantilever length is $l$. The distance from the fixed end to a point on the neutral plane is $s$. The displacement in the $x$-direction at the point $s$ is $u$, and the displacement in the $z$-direction at the point $s$ is $w$. A piezoelectric material at the fixed end can give a displacement input in the $z$-direction, which is set as the control input.

The kinetic energy and the potential energy can be described as

$$
\begin{aligned}
& T=\frac{1}{2} \rho A\left\{\left(\frac{\partial u}{\partial t}\right)^{2}+\left(\frac{\partial w}{\partial t}+\frac{\partial \zeta}{\partial t}\right)^{2}\right\} \\
& V=\frac{1}{2} E I\left(\frac{\partial^{2} w}{\partial s^{2}}+\frac{\partial u}{\partial s} \frac{\partial^{2} w}{\partial s^{2}}-\frac{\partial^{2} u}{\partial s^{2}} \frac{\partial w}{\partial s}\right)^{2}+O\left(w^{4}\right)
\end{aligned}
$$

where $\rho, A, E$, and $I$ respectively denote the density, cross-sectional area, Young's modulus, and geometrical moment of inertia. Here we apply the Lagrange multiplier $\lambda$ under the condition in which the neutral plane is not extended by bending: 


$$
F\left(\frac{\partial u}{\partial s}, \frac{\partial w}{\partial s}\right)=1-\left(\frac{\partial w}{\partial s}\right)^{2}-\left(1+\frac{\partial u}{\partial s}\right)^{2}=0
$$

The extended Hamilton's principle can be written as shown below.

$$
\delta \int_{t_{1}}^{t_{2}} \int_{0}^{l}\left(T-V+\lambda F\left(\frac{\partial u}{\partial s}, \frac{\partial w}{\partial s}\right)\right) d s d t=0
$$

Applying a variational principle to the above equation yields the following $\operatorname{EOM}^{(11)}$.

$$
\begin{aligned}
& \rho A\left(\frac{\partial^{2} w}{\partial t^{2}}+\frac{\partial^{2} \zeta}{\partial t^{2}}\right)+E I \frac{\partial^{4} w}{\partial s^{4}}+C_{\text {lin }} \frac{\partial w}{\partial t} \\
& +E I\left\{\frac{\partial^{4} w}{\partial s^{4}}\left(\frac{\partial w}{\partial s}\right)^{2}+4 \frac{\partial w}{\partial s} \frac{\partial^{2} w}{\partial s^{2}} \frac{\partial^{3} w}{\partial s^{3}}+\left(\frac{\partial^{2} w}{\partial s^{2}}\right)^{3}\right\} \\
& +\frac{\partial}{\partial s}\left[\rho A \frac{\partial w}{\partial s} \int_{l}^{s} \int_{0}^{s}\left\{\left(\frac{\partial^{2} w}{\partial s \partial t}\right)^{2}+\frac{\partial w}{\partial s} \frac{\partial^{3} w}{\partial s \partial t^{2}}\right\} d s d s\right] \\
& =0
\end{aligned}
$$

The associated boundary conditions are as shown below.

$$
\left.\frac{\partial^{3} w}{\partial s^{3}}\right|_{s=l}=\left.\frac{\partial^{2} w}{\partial s^{2}}\right|_{s=l}=\left.\frac{\partial w}{\partial s}\right|_{s=0}=\left.w\right|_{s=0}=0
$$

Effects of the linear viscous damping term $C_{\text {lin }}(\partial w / \partial t)$ are under consideration. The excitation input can be expressed using the piezoelectric constant $d_{33}$ and the input voltage $V_{c}$ as follows (12).

$$
\zeta=d_{33} V_{c}
$$

Furthermore, the slope velocity of the cantilever is feedbacked and the input voltage $V_{c}$ is set such that van der Pol-type self-excited oscillation is realized.

$$
V_{c}=\left.K_{l i n} \frac{\partial^{2} w}{\partial s \partial t}\right|_{s=l}-\left.K_{n o n}\left(\left.\frac{\partial w}{\partial s}\right|_{s=l}\right)^{2} \frac{\partial^{2} w}{\partial s \partial t}\right|_{s=l}
$$

Therein, $K_{\text {lin }}(>0)$ and $K_{n o n}(>0)$ respectively designate the linear feedback gain and the nonlinear feedback gain.

The representative length is set to $l$ and the representative time is set to $T=\sqrt{\rho A l^{4} / E I}$; actually, EOM (5) can be nondimensionalized as follows.

$$
\begin{aligned}
\ddot{w}^{*}+ & w^{*^{\prime \prime \prime \prime}}+\mu_{\text {lin }} \dot{w}^{*} \\
+ & w^{*^{\prime \prime \prime \prime}} w^{*^{\prime} 2}+4 w^{*^{\prime}} w^{*^{\prime \prime}} w^{*^{\prime \prime \prime}}+w^{*^{\prime \prime} 3} \\
+ & {\left[w^{*^{\prime}} \int_{1}^{s^{*}} \int_{0}^{s^{*}}\left(\dot{w}^{*^{\prime 2}}+w^{*^{\prime}} \ddot{w}^{*^{\prime}}\right) d s^{*} d s^{*}\right]^{\prime} } \\
= & -\left.k_{\text {lin }}^{*} \dddot{w}^{\prime *}\right|_{s^{*}=1}+k_{\text {non }}^{*}\left(\left.2 \dot{w}^{\prime * 3}\right|_{s^{*}=1}\right. \\
& \left.+\left.\left.\left.6 w^{\prime *}\right|_{s^{*}=1} \dot{w}^{\prime *}\right|_{s^{*}=1} \ddot{w}^{\prime *}\right|_{s^{*}=1}+\left.\left.w^{\prime * 2}\right|_{s^{*}=1} \dddot{w}^{\prime *}\right|_{s^{*}=1}\right)
\end{aligned}
$$

Here the boundary conditions are written as

$$
\left.w^{*^{\prime \prime \prime}}\right|_{s^{*}=1}=\left.w^{*^{\prime \prime}}\right|_{s^{*}=1}=\left.w^{*^{\prime}}\right|_{s^{*}=0}=\left.w^{*}\right|_{s^{*}=0}=0
$$

where ( ' $)$ denotes a partial derivative with respect to the nondimensional coordinate $s^{*}$ and $\left({ }^{*}\right)$ denotes a partial derivative with respect to nondimensional time $t^{*}$. The dimensionless damping coefficient and the dimensionless feedback gains are set as follows.

$$
\begin{aligned}
& \mu_{\text {lin }}=\frac{C_{\text {lin }} l^{2}}{\sqrt{\rho A E I}} \\
& k_{\text {lin }}{ }^{*}=\frac{d_{33}}{l^{3}} \sqrt{\frac{E I}{\rho A}} K_{\text {lin }} \\
& k_{\text {non }}{ }^{*}=\frac{d_{33}}{l^{3}} \sqrt{\frac{E I}{\rho A}} K_{\text {non }}
\end{aligned}
$$


Subsequently, (*), which indicates the dimensionless quantity, is omitted.

\subsection{Analysis using the method of multiple scales}

The dimensionless EOM (9) with boundary conditions (10) is analyzed using the method of multiple scales. With a small parameter $\epsilon(0<\epsilon \ll 1)$, the $z$-directional displacement $w$ can be expanded into a power series, and multiple time scales are introduced as follows.

$$
\begin{aligned}
& w=\epsilon w_{1}+\epsilon^{3} w_{3} \\
& t_{0}=t, t_{2}=\epsilon^{2} t
\end{aligned}
$$

Each coefficient is given the order shown below so that feedback effects are reflected on the amplitude equation, which is obtained later ${ }^{(13)}$, as

$$
\mu_{\text {lin }}=\epsilon^{2} \hat{\mu}_{\text {lin }}, k_{\text {lin }}=\epsilon^{2} \hat{k}_{\text {lin }}, k_{\text {non }}=\hat{k}_{\text {non }}
$$

where the parameters with $\left(^{\wedge}\right)$ are $O(1)$. The derivatives with respect to $t$ can be expressed in the multiple time scales, as shown below.

$$
\begin{aligned}
& \frac{d}{d t}=\frac{\partial}{\partial t_{0}}+\epsilon^{2} \frac{\partial}{\partial t_{2}} \\
& \frac{d^{2}}{d t^{2}}=\frac{\partial^{2}}{\partial t_{0}^{2}}+2 \epsilon^{2} \frac{\partial^{2}}{\partial t_{0} t_{2}}
\end{aligned}
$$

Subsequently, differential operators $\partial / \partial t_{0} \equiv D_{0}, \partial / \partial t_{2} \equiv D_{2}$ are introduced. Substituting eqs. (14) and (15) into eq. (9) and then equating the coefficients of like powers of $\epsilon$, we obtain the following.

$$
\begin{aligned}
& O(\epsilon): \\
& D_{0}^{2} w_{1}+w_{1}^{\prime \prime \prime \prime}= 0 \\
& O\left(\epsilon^{3}\right): \\
& D_{0}^{2} w_{3}+w_{3}^{\prime \prime \prime \prime}=-2 D_{0} D_{2} w_{1}-\hat{\mu}_{l i n} D_{0} w_{1} \\
&-w_{1}^{\prime \prime \prime \prime} w_{1}^{\prime 2}-4 w_{1}^{\prime} w_{1}^{\prime \prime} w_{1}^{\prime \prime \prime}-w_{1}^{\prime \prime 3} \\
&-w_{1}^{\prime \prime} \int_{1}^{s} \int_{0}^{s}\left\{D_{0}^{2} w_{1}^{\prime 2}+w_{1}^{\prime} D_{0}^{2} w_{1}^{\prime}\right\} d s d s \\
&-w_{1}^{\prime} \int_{1}^{s}\left\{D_{0}^{2} w_{1}^{\prime 2}+w_{1}^{\prime} D_{0}^{2} w_{1}^{\prime}\right\} d s \\
&-\left.\hat{k}_{\text {lin }} D_{0}{ }^{3} w_{1}^{\prime}\right|_{s=1}+\hat{k}_{\text {non }}\left\{2\left(\left.D_{0} w_{1}^{\prime}\right|_{s=1}\right)^{3}\right. \\
&+\left.6 w_{1}^{\prime}\right|_{s=1}\left(\left.D_{0} w_{1}^{\prime}\right|_{s=1}\right)\left(\left.D_{0}^{2} w_{1}^{\prime}\right|_{s=1}\right) \\
&\left.+\left.\left.w_{1}^{\prime 2}\right|_{s=1} D_{0}^{3} w_{1}^{\prime}\right|_{s=1}\right\}
\end{aligned}
$$

Using eq. (16), the approximate solution of $O(\epsilon)$ can be derived as

$$
w_{1}=\Phi_{1}(s) A\left(t_{2}\right) e^{i \omega t_{0}}+C . C .
$$

where the complex amplitude $A\left(t_{2}\right)$ is a function of time scale $t_{2}$. In the approximate solution of $O\left(\epsilon^{3}\right)$, the term with the first eigenfrequency $\omega$ is set as shown in the following.

$$
w_{3}=\Phi_{3}\left(s, t_{2}\right) e^{i \omega t_{0}}+C . C .
$$

Substituting eqs. (18) and (19) into eq. (17) and equating the coefficients of $e^{i \omega t_{0}}$ on both sides of eq. (17) produce the following.

$$
\begin{aligned}
\Phi_{3}^{\prime \prime \prime \prime}-\omega^{2} \Phi_{3}= & -2 i \omega \Phi_{1} D_{2} A\left(t_{2}\right)+C(s)|A|^{2} A \\
& -i \hat{\mu}_{l i n} \omega \Phi_{1} A+i \omega^{3}\left\{\left.\hat{k}_{l i n} \Phi_{1}^{\prime}\right|_{s=1} A-\left.\hat{k}_{n o n} \Phi_{1}^{\prime 3}\right|_{s=1}|A|^{2} A\right\}
\end{aligned}
$$


Here, $C(s)$ can be expressed.

$$
\begin{aligned}
C(s)= & -3 \Phi_{1}^{\prime \prime \prime \prime} \Phi_{1}^{\prime 2}-12 \Phi_{1}^{\prime} \Phi_{1}^{\prime \prime} \Phi_{1}^{\prime \prime \prime}+3 \Phi_{1}^{\prime \prime 3} \\
& +2 \omega^{2} \Phi_{1}^{\prime \prime} \int_{1}^{s} \int_{0}^{s} \Phi_{1}^{\prime 2} d s d s+2 \omega^{2} \Phi_{1}^{\prime} \int_{1}^{s} \Phi_{1}^{\prime 2} d s
\end{aligned}
$$

The condition in which $\Phi_{3}$ can have a solution (solvability condition) is obtainable using $\Phi_{1}{ }^{(7)}$. Multiplying both sides of eq. (20) by $\Phi_{1}$ and integrating it from 0 to 1 with respect to $s$ yields the following.

$$
\begin{aligned}
D_{2} A & +\frac{\hat{\mu}_{\text {lin }}}{2} A+i \beta_{1}|A|^{2} A \\
& -\beta_{2} \hat{k}_{\text {lin }} A+\beta_{3} \hat{k}_{\text {non }}|A|^{2} A=0
\end{aligned}
$$

Therein, $\beta_{1}, \beta_{2}$, and $\beta_{3}$ are as shown below.

$$
\begin{aligned}
& \beta_{1}=\frac{\int_{0}^{1} C(s) \Phi_{1} d s}{2 \omega \int_{0}^{1} \Phi_{1}^{2} d s} \\
& \beta_{2}=\frac{\left.\omega^{2} \Phi_{1}^{\prime}\right|_{s=1} \int_{0}^{1} \Phi_{1} d s}{2 \int_{0}^{1} \Phi_{1}^{2} d s} \\
& \beta_{3}=\frac{\left.\omega^{2} \Phi_{1}^{\prime 3}\right|_{s=1} \int_{0}^{1} \Phi_{1} d s}{2 \int_{0}^{1} \Phi_{1}^{2} d s}
\end{aligned}
$$

Substituting the following equation into eq. (22),

$$
A=\frac{1}{2 \epsilon} a(t) e^{-i \gamma(t)}
$$

and multiplying both sides of it by $\epsilon^{3}$, and subsequently separating it into a real part and an imaginary part yield the following equations for $a$ and $\gamma$.

$$
\begin{aligned}
& \frac{d a}{d t}+\frac{\mu_{\text {lin }}}{2} a-\beta_{2} k_{\text {lin }} a+\frac{1}{4} \beta_{3} k_{\text {non }} a^{3}=0 \\
& \frac{d \gamma}{d t}-\frac{1}{4} \beta_{1} a^{2}=0
\end{aligned}
$$

The equations presented above are equivalent to the equations that govern the time evolutions of the amplitude and the phase of a van der Pol-type self-excited oscillator. Therefore, with the feedback described by eq. (8), the cantilever has the equivalent characteristics to those of a van der Pol-type self-excited oscillator. The steady-state amplitude is obtainable as follows by setting the time-derivative term in eq. (27) to zero.

$$
a_{s t}=0, \sqrt{\frac{-2\left(\mu_{\text {lin }}-2 \beta_{2} k_{l i n}\right)}{\beta_{3} k_{\text {non }}}}
$$

From this, the relationship between the linear feedback gain $k_{\text {lin }}$ and the steady-state amplitude can be shown in Fig. 2. The critical value of linear feedback gain for self-excited oscillation is $k_{\text {lin-cr }}=\mu_{\text {lin }} / 2 \beta_{2}$. The cantilever generates self-excited oscillation by supercritical Hopf bifurcation when the linear feedback gain $k_{\text {lin }}$ is greater than this critical value $k_{\text {lin-cr }}$. On the other hand, the fact that the nonlinear feedback gain $k_{n o n}$ is included in the denominator in eq. (29) means that the steady-state amplitude of the cantilever in self-excited oscillation can be suppressed sufficiently by enlarging $k_{n o n}$. Furthermore, by solving eq. (28), the following equation is derived.

$$
\gamma=\frac{1}{4} \beta_{1} a_{s t}^{2} t+\gamma_{0}
$$

From this, the phase of the cantilever vibration is obtainable. Here, $\gamma_{0}$ is the initial phase, which is determined by the initial condition. Consequently, the steady state of the self-excited oscillation of the cantilever can be written as shown below.

$$
v=a_{s t} \cos \left\{\left(\omega-\frac{1}{4} \beta_{1} a_{s t}^{2}\right) t-\gamma_{0}\right\}
$$




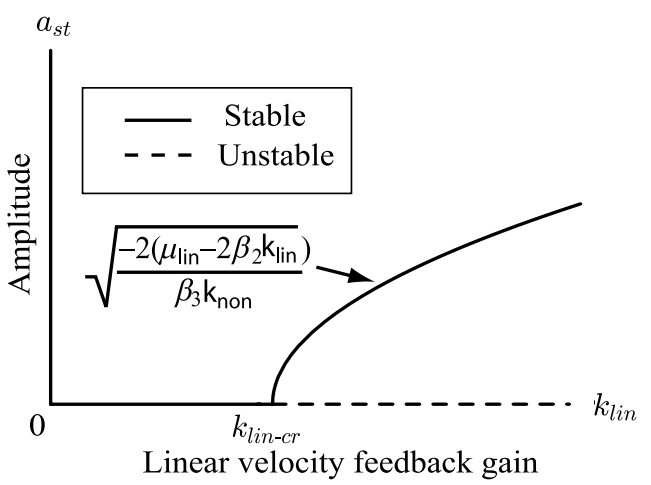

Fig. 2 Bifurcation diagram (Relationship between amplitude and linear velocity feedback gain)

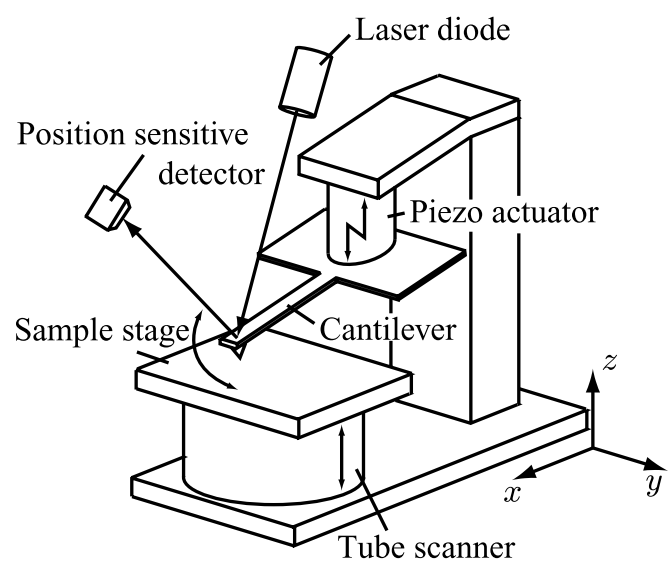

Fig. 3 Setup of the AFM prototype

Therein, $\omega$ is the linear eigenfrequency of the cantilever. The self-excited oscillation frequency of the cantilever $\Omega$ is

$$
\Omega=\omega-\frac{1}{4} \beta_{1} a_{s t}^{2}
$$

Typically, $\Omega$ and $\omega$ are almost equal when the steady-state amplitude $a_{s t}$ is small.

\section{Prototyped AFM for experiments}

\subsection{Configuration of the AFM equipment}

For this study, we produced an AFM prototype, as shown in Figs. 3 and 4, to conduct experiments. Compared to a commercially available AFM, the prototyped AFM has advantages that include its more efficient parameter study in experiments and its easier addition of measuring instruments to observe the behavior of each mechanical element commencing with the microcantilever.

A tube scanner is installed under the sample stage. It can conduct scanning in $x-y$ directions and control the position in the z-direction for the sample. The cantilever is excited at its support part by the piezo-ceramic actuator. The optical lever method ${ }^{(14)}$, in which the top surface of the cantilever is lased and the reflection angle of the laser beam is detected, is applied to measure the cantilever slope.

In subsequent sections of this paper, the optical lever-type slope detection mechanism, the cantilever holder, and the feedback circuits that are used to realize the applied methods are explained in greater detail.

\subsection{Slope detection apparatus using optical lever method}

Figure 5 shows a schematic diagram of the slope measuring mechanism using the optical lever method. A laser beam from the laser pointer (MLXG-D12-670-3; Kikoh Giken) is 


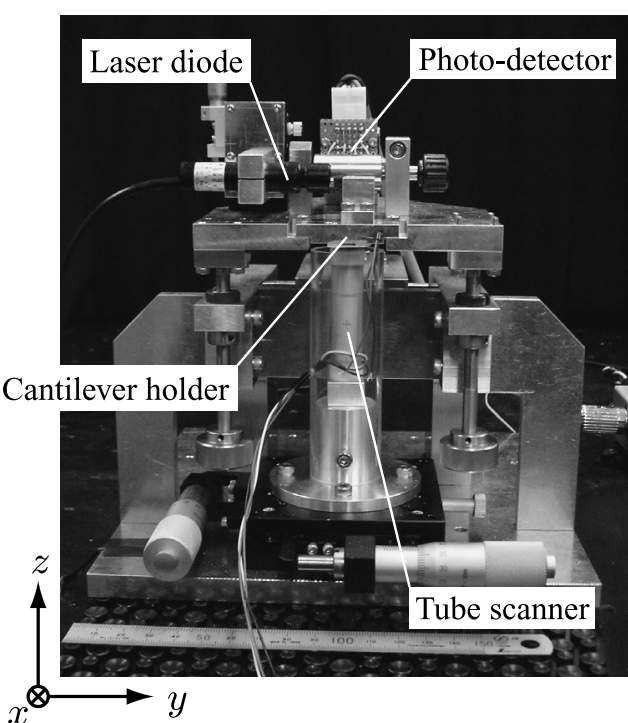

Fig. 4 Photograph of the AFM prototype

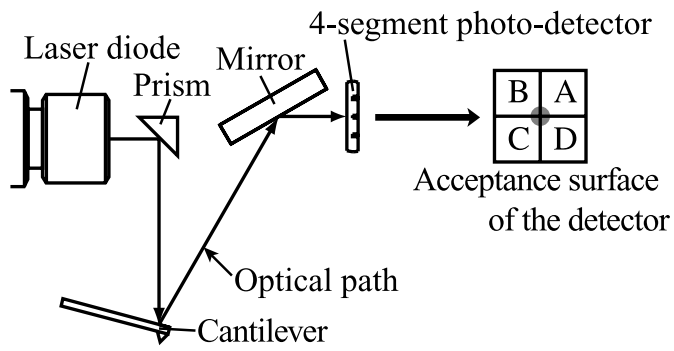

Fig. 5 Acceptance surface of a four-segment photo-detector

projected at the upper surface of the free end of the cantilever. Then, the reflecting laser beam is received by the four-segmental photo-detector (S7479; Hamamatsu Photonics KK) on contact with the mirror. The reflection angle varies as the cantilever bends. Consequently, the light spot of the reflected laser beam moves. The cantilever slope can therefore be measured using the change in the photo-acceptance position.

Figure 5 shows that the photo-acceptance position is detectable as the difference between the light amount, which is received by two segments in the upper half of the four segments, and one which is received by two segments in the lower half of the four segments. As preparations preceding an observation, two adjustments are necessary. One is adjustment such that the laser beam is directed at the top of the upper surface of the cantilever. The other is adjustment such that the reflecting beam is received at the center of the four-segmental photo-detector in the stationary state. For these purposes, the laser pointer is mounted on the stage (TBM254CL; Sigma Koki Co. Ltd.) to enable adjustment of the irradiated point. The laser pointer is fixed to keep it horizontal, the laser beam is diverted downward vertically through the prism (CSCH-10-550; Sigma Koki Co. Ltd.) and is directed at the cantilever. Because the cantilever is supported at an angle of $15 \mathrm{deg}$ to the horizontal plane, the reflecting beam is reflected at an angle of about $30 \mathrm{deg}$ to the perpendicular direction and heads back to the mirror. The rough adjustment that enables the reflecting beam to be received by the photo-detector is carried out using the mirror (TFA-10S03-10; Sigma Koki Co. Ltd.), which can adjust its angle. Moreover, the photo-detector is fixed to the stage (TASB-252; Sigma Koki Co. Ltd.) to position the device precisely.

Each segment of the photo-detector outputs electric current in direct relation to each amount of received light. Each electric current is converted into an electric voltage by the I-V converter circuit shown in Fig. 7; then the difference in the amount between light received by the upper two segments and one received by the lower two segments is calculated using the 


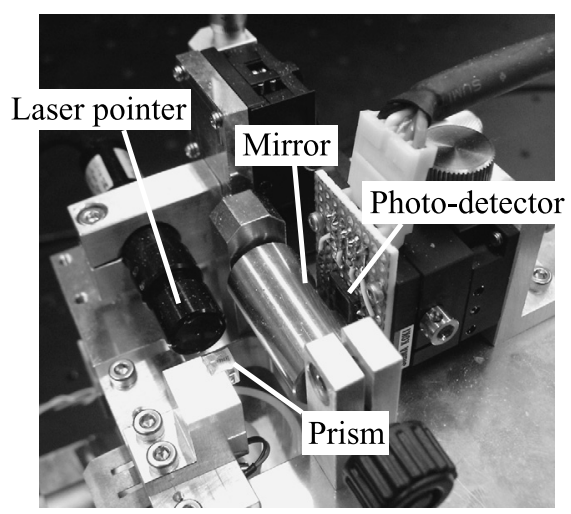

Fig. 6 Photograph of the optical lever system

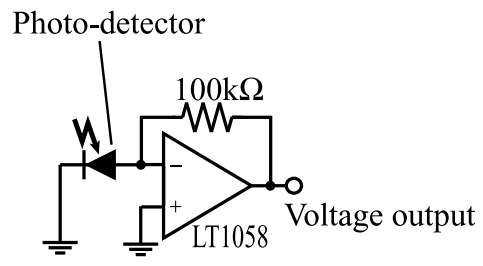

Fig. 7 I-V converter circuit

arithmetic circuit depicted in Fig. 8. The operational amplifier (LT1058CN; Linear Technology), of which the bias current is small, is used as the I-V converter; the precise operational amplifiers (NE5532, NE5534; Texas Instruments) are used in the arithmetic circuit. The electrical circuit, which can obtain the sum of outputs from all four segments, is incorporated into the arithmetic circuit so that the amount of light received by the entire photo-detector is measurable.

\subsection{Cantilever holder}

The configuration and image of the cantilever holder are shown, respectively, in Figs. 9 and 10. The cantilever is supported at an angle of about $15 \mathrm{deg}$ from the horizontal direction. The piezoelectric element (Fuji Ceramics Corp.) is glued on the upper part of the supporting platform to impart a displacement excitation input to the cantilever. Because the cantilever is fixed by being clipped by a plate spring made of phosphor bronze, it is easy to exchange.

\subsection{Electrical circuit for van der Pol type self-excited oscillation}

In this study, the cantilever is made into a vibratory system equivalent to a van der Poltype self-excited oscillatory system using linear feedback and nonlinear feedback. The electrical circuit shown in Fig. 12 is manufactured so that the linear feedback and the nonlinear feedback as expressed by eq. (8), which is in proportion to the squared slope and the slope velocity, are realized. The signal of the slope measured using the optical lever device is differentiated with respect to time and is amplified by the electrical circuit so that the linear

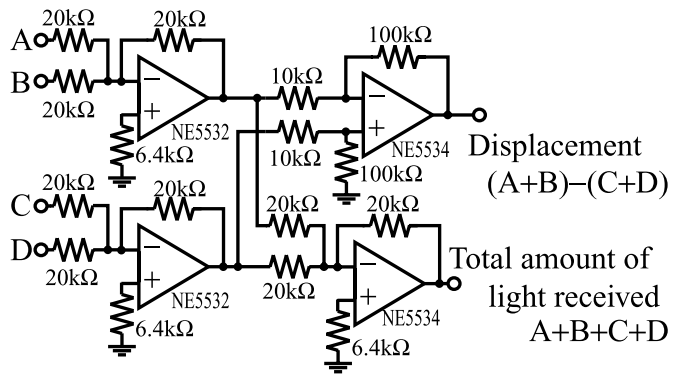

Fig. 8 Slope signal processor circuit 


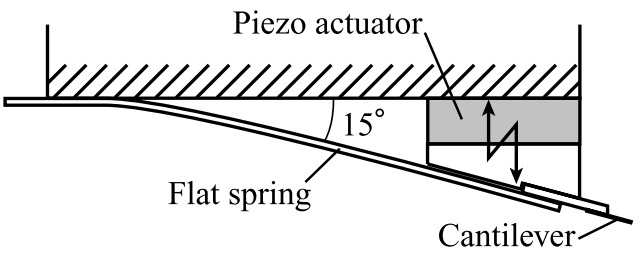

Fig. 9 Setup of the cantilever holder

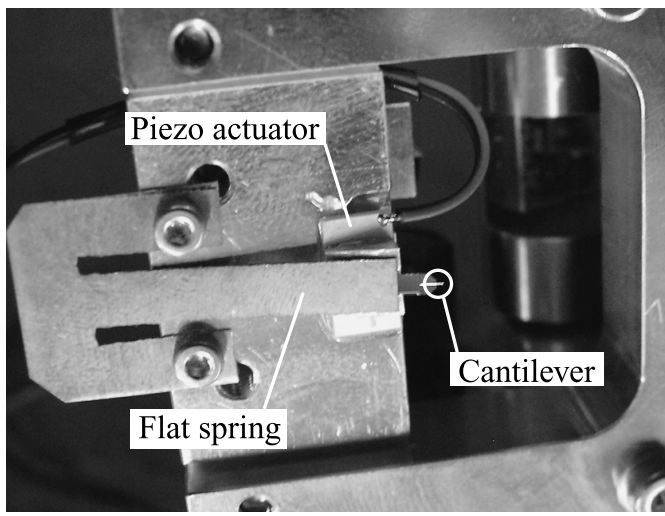

Fig. 10 Photograph of the cantilever holder

feedback signal is produced. The nonlinear feedback signal comprises the slope signal and slope-velocity signal, which is obtained from differentiating the slope signal with respect to time, using the analog multiplier (AD734; Analog Devices). Finally, the linear component and nonlinear component are added and the result is impressed to the piezoelectric element as the control input for the cantilever. The variable resisters can adjust the linear feedback gain and the nonlinear feedback gain separately.

\section{Experiments using the AFM prototype}

Experiments were performed using the AFM prototype installed with the cantilever with $450 \mu \mathrm{m}$ length, $50 \mu \mathrm{m}$ width, $4 \mu \mathrm{m}$ thickness and $27.9 \mathrm{kHz}$ as the first natural frequency (SI-DF3; Seiko Instruments Inc.), as shown in Fig. 11.

\subsection{Realization of self-excited oscillation using the optical lever method}

First, the cantilever was forced to generate self-excited oscillation using only linear feedback. At that time, the cantilever generated self-excited oscillation with the natural frequency of the second vibration mode, which can occur because the output from the optical lever method, which detects the slope of the cantilever, has higher sensitivity for the second vibration mode than that for the first vibration mode. This study is intended to show that self-

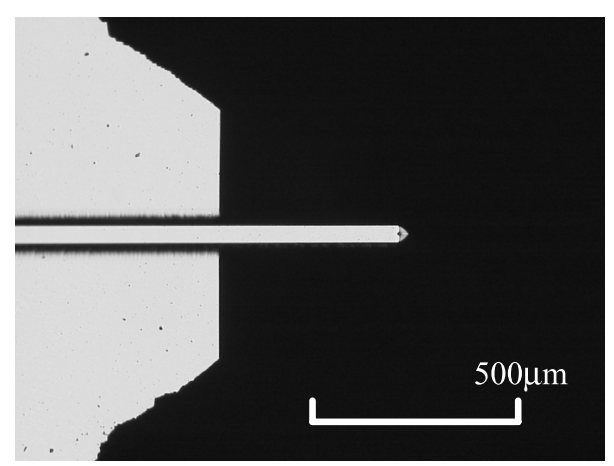

Fig. 11 Photograph of the microcantilever 


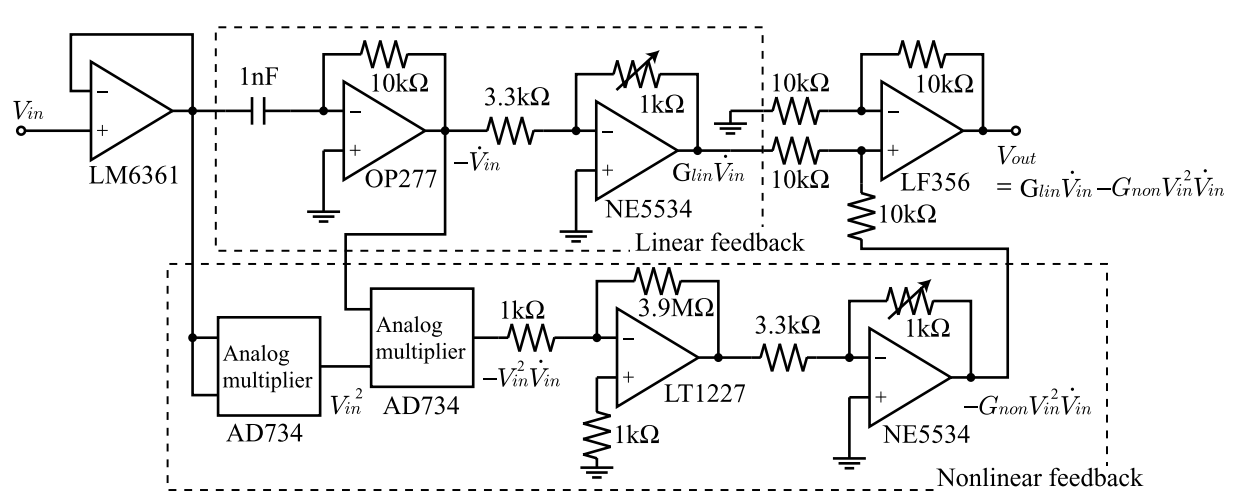

Fig. 12 Electronic circuit for "van der Pol" type self-excitation

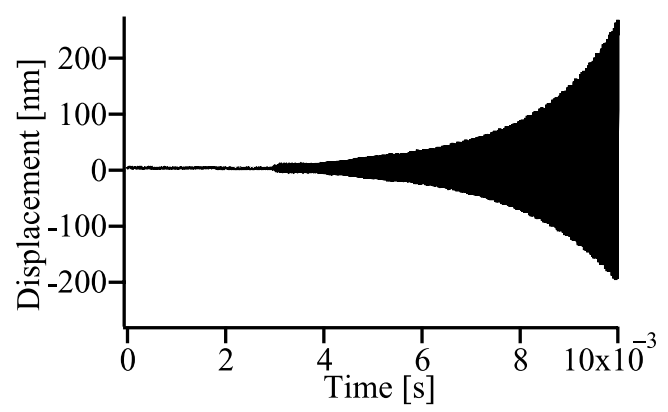

Fig. 13 Time history in the case of only linear velocity feedback

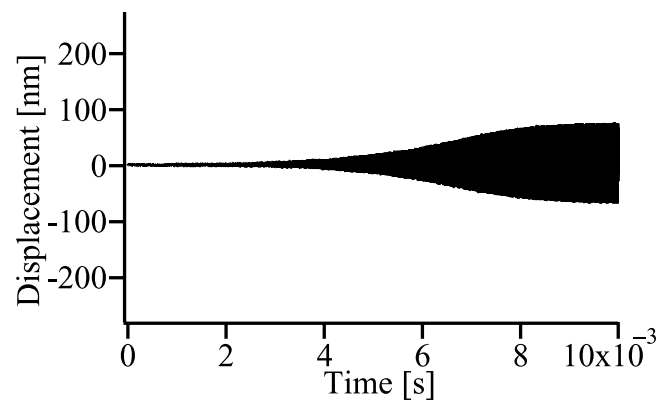

Fig. 14 Time history in the case of linear and nonlinear velocity feedback

excited oscillation is realized at the natural frequency of the first vibration mode, which is utilized more often in AFM. The laser is therefore focused at a location which is about 220 $\mu \mathrm{m}$ distant from the fixed end of the cantilever, where the output of the second vibration mode becomes small in the output from the optical lever, to lower the sensitivity for the second vibration mode. Consequently, the cantilever generates self-excited oscillation at the natural frequency of the first vibration mode.

\subsection{Achievement of self-excited oscillation with steady and small amplitude}

The time history of self-excited vibration is portrayed in Fig. 13 for the case in which only the linear feedback is applied. The amplitude of the self-excited oscillation by the linear feedback grows and diverges as time passes. Additionally, an amplitude greater than $250 \mathrm{~nm}$ can not be measured because of the sensor limitations.

Next, based on the method proposed conceptually in the second chapter, the nonlinear feedback is added to control the amplitude of the self-excited oscillation. A time history in the case that the nonlinear feedback is added to the linear feedback is shown in Fig. 14. The growth of the amplitude falls within a certain constant magnitude; the cantilever vibrates with a steady-state amplitude afterward, whereas the amplitude grows and diverges only by the linear feedback. Figure 15 depicts the relationship between the magnitude of the steady-state 


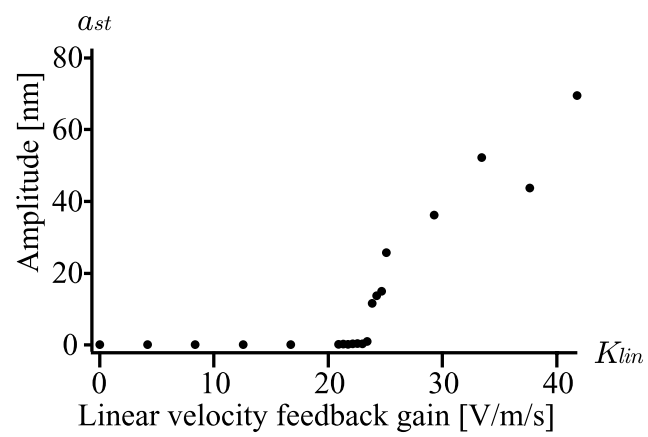

(a) Nonlinear feedback gain $K_{\text {non }}=1.8 \times 10^{16} \mathrm{~V} / \mathrm{m}^{3} / \mathrm{s}$

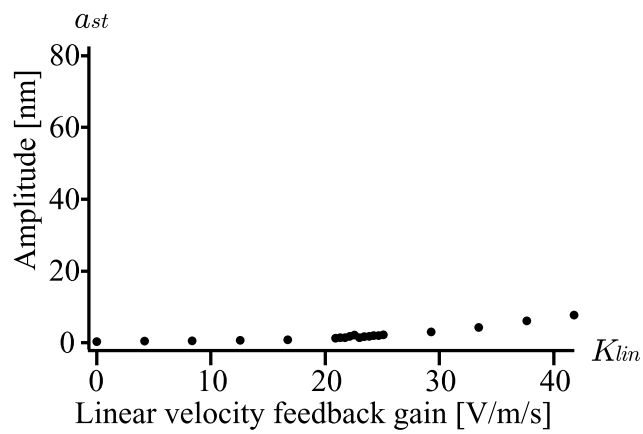

(b) Nonlinear feedback gain $K_{\text {non }}=3.6 \times 10^{17} \mathrm{~V} / \mathrm{m}^{3} / \mathrm{s}$

Fig. 15 Relationship between the amplitude and the linear velocity feedback gain $K_{\text {lin }}$

amplitude of the self-excited oscillation and the linear feedback gain. The horizontal axis shows the linear feedback gain, $K_{l i n}$, and the vertical axis shows the steady-state amplitude of the self-excited oscillation, $a_{s t}$. The self-excited vibration takes place in the range in which the linear feedback gain $K_{\text {lin }}$ is greater than the critical gain, $K_{\text {lin-cr }}$. Without the nonlinear feedback, the vibration amplitude reaches a limitation in measurement immediately after the linear feedback gain becomes greater than the critical gain. The amplitude is controlled by the effect of the nonlinear feedback, as shown in Fig. 15(a). Moreover, the amplitude is suppressed using a greater nonlinear feedback gain $K_{\text {non }}$, as shown in Fig. 15(b). In addition, self-excited oscillation with $8 \mathrm{~nm}$ steady-state amplitude can be confirmed experimentally in this case.

It is noticed that a small-amplitude self-excited oscillation can be achieved theoretically by setting the linear feedback gain $K_{\text {lin }}$ to be very close to the critical gain $K_{\text {lin-cr }}$. In practice, however, $K_{\text {lin-cr }}$ (bifurcation point) varies easily according to small changes in the environment. As a result, the self-excited oscillation often stops. Using the technique proposed in this study, the value of the linear feedback gain can be configured to be a sufficiently larger value than $K_{\text {lin-cr }}$. Even in such a case, the amplitude can be suppressed sufficiently. Therefore, a stable and small amplitude steady-state oscillation can be achieved without stopping the oscillation.

\section{Conclusions}

Toward realizing observation of a biological sample in liquid using AFM, in this paper, a self-excitation method for the AFM microcantilever was proposed. Its feasibility and usefulness were confirmed through experimentation. The results of this study can be summarized as follows.

(1)With linear feedback and nonlinear feedback, the microcantilever can be equipped with dynamics equivalent to those of a van der Pol-type self-excited oscillator. Consequently, steadystate oscillation is realized to achieve sufficient suppression of its amplitude.

(2)The AFM equipment was prototyped. Using it, we performed parameter studies experi- 
mentally to elucidate the bifurcation characteristics of the self-excited oscillation-type microcantilever. The nonlinear characteristics were clarified using experiments.

(3)Self-excited oscillation with the steady-state amplitude of $8 \mathrm{~nm}$ was accomplished using a high-gain nonlinear feedback.

\section{Acknowledgements}

The authors wish to thank Mr. Takashi Someya (Graduate School of System and Information Engineering, University of Tsukuba) for his assistance with experiments. This work was supported by a Grant-in-Aid for Science Research from Japanese Ministry of Education, Culture, Sports, Science and Technology (Basic Research (C), No. 16560377 and 19560225).

\section{References}

( 1 ) Morita, S., Wiesendanger, R., and Meyer, E. (Eds.), Noncontact Atomic Force Microscopy, (2002), Springer, Berlin.

( 2 ) Binnig, G., Quate, C. F., and Gerber, C., Atomic Force Microscope, Physical Review Letters. Vol. 56, (1986), pp. 930-933.

( 3 ) Albrecht, T. R., Grutter, P., Horne, D., and Ruger, D., Frequency Modulation Detection Using High-Q Cantilevers for Enhanced Force Microscope Sensitivity, Journal of Applied Physics Vol. 69, (1991), pp. 668-673.

( 4 ) Okajima, T., Sekiguchi, H., Arakawa, H., and Ikai, A., Self-Oscillation Technique for AFM in Liquids, Applied surface science, Vol. 210, (2003), pp. 68-72.

( 5 ) Okajima, T., Tokumoto, H., Versatility of Self-Oscillation Technique with MechanicalAcoustic Excitations for Frequency Modulation Atomic Force Microscope in Liquids, Japanease Journal of Applied physics, Vol. 43, No. 7B, (2004), pp. 4634-4638.

( 6 ) Kaneko, H., Kuroda, M., and Yabuno, H., Nonlinear Analysis of a Self-excited Cantilever Beam, Proceedings of DETC2005, (2005), 84843.

( 7 ) Yabuno, H., Kaneko, H., Kuroda, M, and Kobayashi, T., Van der Pol type self-excited micro-cantilever probe of atomic force microscopy, Nonlinear Dynamics, (in press).

( 8 ) Nayfeh, A. H., Perturbation methods, (1973), Wiley Interscience, New York.

( 9 ) Hayashi, K., Kobayashi, T., Kuroda, M., and Yabuno, H., "Van der Pol" Self-excitated Microcantilever with PZT Actuator and Sensor, Abstracts of the 9th International Conference on Non-contact Atomic Force Microscopy, (2006-7), p. 51.

(10) Kuroda, M. and Yabuno, H., "Cantilever Control Device," Japanese Patent Disclosure 2006-208089 (2006).

(11) Crespo da Silva, M. R. M., and Clynn, C. C., Nonlinear Flexural-flexural-torsional Dynamics of Inextensional Beam-I. Equations of Motion, Journal of Structural Mechanics, Vol. 6, No. 4 (1978), pp. 437-448.

(12) Fuller, C. R., Elliott, S. J., and Nelson, P. A., Active Control of Vibration, (1996), Academic Press Inc..

(13) Yabuno, H., Bifurcation Control of Parametrically Excited Duffing System by a Combined Linear-Plus-Nonlinear Feedback Control, Nonlinear Dynamics, Vol. 12, (1997), pp. 263-274.

(14) Alexander, S., Hellemans, L., Marti, O., Schneir, J., Elings, V., Hansma, P. K., Longmire, M., and Gurley, J., An Atomic-resolution Atomic-force Microscope Implemented Using an Optical lever, Journal of Applied Physics Vol. 65, No. 1 (1989), pp. 164-167. 